\title{
Detection and analysis of wheat storage year based on electronic tongue and DWT-IPSO-LSSVM algorithm
}

\author{
Tingting Guo, Zhengwei Yang, Nan Miao, Xin Zhang, Qingsheng Li, J ie Guo, Zhiqiang Wang* \\ (College of Computer Science and Technology, Shandong University of Technology, Zibo 255049, China)
}

\begin{abstract}
The electronic tongue system based on virtual instrument technology was used to qualitatively analyze the wheat, which achieves rapid and objective evaluation analysis of aged wheat with different storage years. In view of the complex output signal of the electronic tongue and the large amount of data, the Discrete Wavelet Transform was used to extract the eigenvalues of the original data to reduce the data dimension size. On this basis, the improved particle swarm optimization algorithm was used to optimize the parameters of Least squares support vector machine, and the analysis model of wheat storage age was established. The experiments exhibited that the DWT-IPSO-LSSVM model had better classification performances than other pattern recognition models, such as DWT-GA-LSSVM, DWT-AF-LSSVM and DWT-PSO-LSSVM. The results showed that the accuracy of the training set, the accuracy of the test set, overall classification accuracy and Kappa coefficient of the proposed combined model in this paper were $95 \%, 92 \%, 91 \%$ and 0.88 respectively. This research indicated that the electronic tongue system combined with proposed model can be used to identify and discriminate the aged wheat with different storage years.
\end{abstract}

Keywords: electronic tongue, wheat storage year, discrete wavelet transform, improved particle swarm optimization, least squares support vector machine

DOI: $10.33440 /$ j.ijpaa.20190202.46.

Citation: Guo T T, Yang Z W, Miao N, Zhang X, Li Q S, Guo J, Wang Z Q. Detection and analysis of wheat storage year based on electronic tongue and DWT-IPSO-LSSVM algorithm. Int J Precis Agric Aviat, 2019; 2(2): 19-24.

\section{Introduction}

Wheat is one of the main grain reserves in China, and its quality affects people's quality of life and health directly. With the increase of storage time, the stocked wheat gradually ages with the possibility of moldy. The consumption of moldy wheat may cause acute poisoning and a variety of gastrointestinal diseases. At present, the common methods for detecting grain aging degree include sensory detection method, guaiacol reaction method, tetrazolium salt staining method and infrared spectrum analysis method. The sensory evaluation mainly relies on human sensory organs, which is susceptible to external interference and exist the disadvantage of strong subjectivity and poor repeatability ${ }^{[1]}$. The coloration time and color of guaiacol reaction method are difficult to grasp accurately for wheat with similar storage time because of the unobvious difference in color depth ${ }^{[2]}$. The titration endpoint is difficult to judge, which causes the results of different operators are not comparable ${ }^{[3]}$. The infrared spectrum analysis method is expensive, bulky, and inconvenient to carry ${ }^{[4]}$. In general, these methods are difficult to meet the need for rapid on-site inspection of stored wheat. Therefore, it is urgent need to develop and

Received date: 2019-11-28 Accepted date: 2019- 12-15

Biographies: Tingting Guo, Postgraduate student, research interest: Detection and control technology, Email:2504808963@qq.com; Zhengwei Yang, Postgraduate student, research interests: Deep learning, Email:577524343@ qq.com; Nan Miao, Postgraduate student, research interests: Artificial intelligence, Email:969635021@qq.com; Xin Zhang, Postgraduate student, research interests: Deep learning, Email:1210678177@qq.com; Qingsheng Li, Postgraduate student, research interests: Deep learning, Email:378140341@ qq.com; Jie Guo, Postgraduate student, research interests: Embedded system and Application, Email: 502859669@qq.com.

*Corresponding author: Zhiqiang Wang, $\mathrm{PhD}$, professor, research interests: Detection and control technology, Email: wzq@sdut.edu.cn. establish new technologies that are efficient, convenient and achieving on-site testing for aged wheat.

The electronic tongue is a new type of detection instrument which employs multi-sensor array and multivariate statistical analysis method to analyze complex solution. It has the advantages of simple operation, rapid detection, portability, good reproducibility and strong objectivity, etc. In recent years, it has been successfully applied to the analysis and detection of tea ${ }^{[5]}$, water $^{[6]}$, wine ${ }^{[7]}$ and meat ${ }^{[8]}$. Nevertheless, to the best of our knowledge, it has seldom been reported to detect the storage years of wheat. The pattern recognition technology is the key technology for electronic tongue signal processing. This process mainly includes two stages: feature value extraction and classification recognition. At present, the common feature extraction methods mainly include cross-sectional area method, principal component analysis method, Fourier transform and wavelet transform. However, the cross-sectional area method and principal component analysis only extract the finite characterization parameters in the signal, and the extracted information cannot fully reflect the sample information ${ }^{[9,10]}$. The Fourier transform is suitable for feature extraction of stationary signals, which only characterizes time-domain or frequency-domain information ${ }^{[11-12]}$. For the classification recognition method, the common used algorithms mainly include neural network ${ }^{[13]}$, support vector machine ${ }^{[14]}$, random forest ${ }^{[15]}$, extreme learning machine ${ }^{[16]}$, etc. However, these methods are limited by the difficulty of selection parameter.

In this paper, a laboratory-developed electronic tongue system is applied to realize qualitative discrimination and analysis of aged wheat. According to the characteristics of the electronic tongue response signal, the Discrete Wavelet Transform (DWT) is employed to preprocess the electronic tongue signal, and the Improved particle swarm optimization (IPSO) is applied to 
optimize the parameters of the Least squares support vector machine (LSSVM). Finally, a wheat aging detection model was established, which was designed to provide the theoretical basis and technical support for grain storage.

\section{Materials and methods}

\subsection{Experimental materials and sample processing}

The experimental wheat was provided by the national grain reserve in the eastern suburbs of Zibo city, Shandong province of China. The reserve conditions of wheat strictly followed the national standard of China. Five kinds of aged wheat samples were collected in June 2018, which are harvest from 2013 to 2017. The collected samples were selected and screened at room temperature to remove impurities and debris. Before the experiments, five kinds of aged wheat were accurately weighed. Then $30 \mathrm{~g}$ samples were placed in a beaker, soaked in $200 \mathrm{~mL}$ pure water at $100^{\circ} \mathrm{C}$ for 20 minutes, and filtered by filter paper. In order to avoid the influence of electrode surface residue, $\mathrm{Al}_{2} \mathrm{O}_{3}$ grinding powder was used to polish the electrode, and deionized water was used to clean the electrode surface.

\subsection{Electronic tongue system}

The self-developed electronic tongue system is mainly composed of the sensor array, signal conditioning circuit, data acquisition card, and LabVIEW software system. The structure and physical diagram of the electronic tongue system are shown in Figure 1. The virtual machine software controls the data acquisition card to generate an excitation signal, which is applied to the sensor array through the signal conditioning module. The electrochemical reactions occur on the sensor surface and generate a weak current response signal. Then the response current is collected, converted and filtered by the signal conditioning module, and then sent to the data acquisition card for A/D conversion. Finally, the signal is transfer to the computer for signal processing and pattern recognition analysis ${ }^{[17]}$.

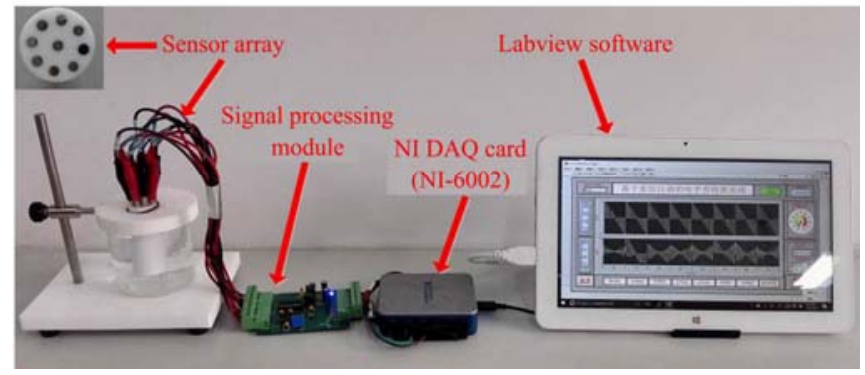

a. Physical picture of electronic tongue

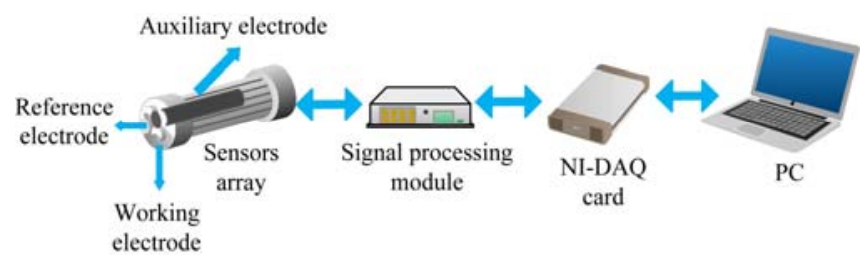

b. The structure diagram

Figure 1 The electronic tongue instrument

\subsection{Data analysis method}

\subsubsection{Discrete Wavelet Transform}

DWT is an efficient, simple and fast joint analysis method in time and frequency domain, which can analyze signals with multi-resolution, adaptive and time-frequency domain localization ${ }^{[18]}$. In this paper, according to the characteristics of the response signal, the Mallat wavelet decomposition is used to analysis the signal of electronic tongue. The formula is as follows:

$$
\left\{\begin{array}{l}
p_{k}^{j}=\sum_{m \in Z} \alpha_{m-2 k} P_{m}^{j-1} \\
r_{k}^{j}=\sum_{m \in Z} \beta_{m-2 k} P_{m}^{j-1}
\end{array}\right.
$$

where, $p_{k}^{j}$ is low frequency components of original signal after Wavelet decomposition; $r_{k}^{j}$ is the high frequency component of the original signal after wavelet decomposition; $\alpha$ and $\beta$ are low-pass filter and high-pass filter respectively; $\alpha_{m-2 k}$ and $\beta_{m-2 k}$ are low-pass filter coefficients and high-pass filter coefficients respectively; $j$ is the number of decomposed layers. After wavelet decomposition, the high-frequency components in the signal are removed. Only the low-frequency components are retained, which does not cause the loss of valid information in the original signal. Therefore, the electronic tongue data can be efficiently compressed and feature extracted.

The inverse reconstruction of the Mallat reconstruction algorithm can obtain the compressed reconstructed signal. This signal maintains the basic waveform characteristics and effective information compared with the original signal. The Mallat reconstruction algorithm is as follows:

$$
P_{k}^{j-1}=\sum_{m \in Z} \alpha_{k-2 m}^{\prime} p_{m}^{j}+\sum_{m \in Z} \beta_{k-2 m}^{\prime} r_{m}^{j}
$$

Mother wavelets and decomposition level are two important parameters that influenced the compression effect of DWT. Different mother wavelets have different time-domain and frequency-domain features, which extract different feature information from the original signal. The number of decomposition layers determines the size of the final data and affects the classification performance of the model ${ }^{[19]}$. For optimizing the number of mother wavelet and wavelet decomposition layers, the similarity coefficient $\mathrm{R}$ is introduced to evaluate the compression effect of DWT. The calculation formula of similarity coefficient $R$ is:

$$
R=\frac{\operatorname{cov}(i, j)}{\sqrt{\operatorname{cov}(i, i) \cdot \operatorname{cov}(j, j)}}
$$

where, $i$ is original signal data point, $\mathrm{j}$ is reconstruction of signal data points by DWT compression, $\operatorname{cov}(i, j)$ represents covariance for two sets of signals. The larger the correlation coefficient is, the compressed and reconstructed signal is closer to the original signal, which means the information extraction effect is better.

2.3.2 Least squares support vector machine

Support vector machine (SVM) is a supervised machine learning method based on statistical learning theory ${ }^{[20]}$. Least squares support vector machine (LSSVM) is a new improved model for support vector machine, which is based on the principle of structural risk minimization. It uses the least squares linear system to solve the regression classification problem, which reduces the computational complexity and improves the solution speed. By changing the empirical risk from the first power to the second power, and replacing the inequality constraints with equality constraints, the LSSVM can be described as:

$$
\min J(\omega, b, e)=\frac{1}{2} \omega^{T} \omega+\gamma \frac{1}{2} \sum_{i=1}^{n} e_{i}^{2}
$$

where, $\omega$ is the weight vector; $\gamma$ is the regularization parameter; $e_{i}$ is the error vector, and $b$ is the offset.

The prediction function of LSSVM is:

$$
y(x)=\sum_{i=1}^{l} a_{i} K\left(x, x_{i}\right)+b
$$


Different kernel functions $K\left(x_{i} \cdot x\right)$ in LSSVM can implement different algorithms. Currently, the common used kernel functions include polynomial kernel function, Sigmoid kernel function and radial basis kernel function. Due to good applicability of radial basis kernel function to low-dimensional and high-dimensional data, this study uses radial basis kernel as kernel function of LSSVM model ${ }^{[21]}$. The radial basis kernel can be expressed as:

$$
K=\frac{N \sum_{k=1}^{N} a_{k k}-\sum_{i=1}^{N} a_{k i} a_{i k}}{N^{2}-\sum_{i=1}^{N} a_{k i} a_{i k}}
$$

where, $X$ is input variable; $X_{i}$ is center point of RBF function; $\sigma$ is the Kernel function parameter.

The penalty factor $\mathrm{C}$ and the kernel parameter $\sigma$ are two important factors that affect the predictive power and generalization ability of the LSSVM model ${ }^{[22]}$. The penalty factor C can achieve the balance between minimizing the training error and the model complexity. The kernel parameter $\sigma$ determines the nonlinear mapping from the sample space to the high-dimensional feature space. Therefore, in order to improve the accuracy of the model, it is necessary to optimize the penalty factor and kernel parameter of the LSSVM.

\subsubsection{The model of IPSO-LSSVM}

In this paper, the improved particle swarm optimization (IPSO) is used to optimize $\sigma$ and $C$ of LSSVM, which can reduce the blindness of subjective experience and improve its prediction performance to a large extent. The vector $X_{i}=\left[x_{i 1}, x_{i 2}, \cdots, x_{i D}\right]^{T}$ represents the position of the $\mathrm{i}$-th particle in the d-dimensional search space, which is a potential solution of the problem. According to the objective function, the fitness corresponding to the position of each particle can be calculated. $v_{i}=\left[v_{i 1}, v_{i 2}, \ldots\right.$, $\left.v_{i D}\right]^{T}$ is the velocity of the i-th particle. The global extremum of the population is $P_{g}=\left[p_{g 1}, p_{g 2}, p_{g D}\right]^{T}$. In the iterative process, the particles update their own speed and position by the individual extreme and global extreme values. The update formula is

$$
\begin{gathered}
v_{i d}^{k+1}=w v_{i d}^{k}+c_{1} r_{1}\left(p_{i d}^{k}-x_{i d}^{k}\right)+c_{2} r_{2}\left(p_{g d}^{k}-x_{i d}^{k}\right) \\
P_{i d}^{k}-x_{i d}^{k}=x_{i d}^{k}+v_{i d}^{k+1} \quad(d=1,2, \cdots, D ; i=1,2, \cdots, n)
\end{gathered}
$$

where, $k$ is the current iteration number; $v_{i d}$ is particle velocity; $c_{1}$, $c_{2}$ is acceleration factor, and $r_{1}, r_{2}$ random number distributed between $[0,1]$.

In the early stages of evolution, particles are expected to have better exploration capabilities. With the increase of the number of iterations, particles are expected to have better development capabilities $^{[23]}$. In the particle swarm algorithm, $c_{1}$ and $c_{2}$ are the self-learning factors and social learning factors, respectively. In this paper, the dynamic adjustment of self-learning factors and social learning factors are adopted, and the specific adjustment strategies are as follows:

$$
\left\{\begin{array}{l}
c_{1}=\left(c_{1 e}-c_{1 s}\right) \frac{k}{k_{\max }}+c_{1 s} \\
c_{2}=\left(c_{2 e}-c_{2 s}\right) \frac{k}{k_{\max }}
\end{array}\right.
$$

where, $c_{1 s}, c_{1 e}, c_{2 s}, c_{2 e}$ are the initial value and final value of $c_{1}$ and $c_{2}$, respectively; $k_{\max }$ is the maximum number of iterations.

The objective function is defined by using IPSO optimization LSSVM.

$$
\min f(C, \sigma)=\sum_{i=1}^{m}\left(x_{i}-\widehat{x}_{i}\right)^{2}\left(C \in\left[C_{\min }, C_{\max }\right], \sigma \in\left[\sigma_{\min }, \sigma_{\max }\right]\right)(10)
$$

where, $x_{i}$ is the output value of the $\mathrm{i}$-th known sample, and $\hat{x}_{i}$ is the model prediction value of the LSSVM for the i-th sample.

The idea of IPSO-LSSVM is to search a set of parameters $(c, \sigma)$ by an iterative algorithm to minimize the objective function of the formula (10). The specific implementation steps are shown in Figure 2, which is as follows:

(1) Input five storage years aging wheat sample data as training sample set.

(2) Set the relevant parameters of the model and initialize the position and velocity of the particles. Each particle corresponds to a set of kernel function parameters $\sigma$ and penalty factor parameters $C$ of the LSSVM in the D-dimensional search space. The LSSVM prediction model is established by the parameters and the training samples input in step (1).

(3) Calculate the fitness value of each particle from Equation (10). Set the fitness function as the mean square error of the model prediction.

(4) Determine whether the termination conditions are met. If condition is satisfied, the iteration ends and the optimal parameters $(c, \sigma)$ are output. Otherwise, continue to step (5).

(5) Update the position and velocity of the particles according to formula (7), formula (8), and formula (9).

(6) The detection model of aged wheat based on IPSO-LSSVM was established by using the optimal parameter combination and training samples.

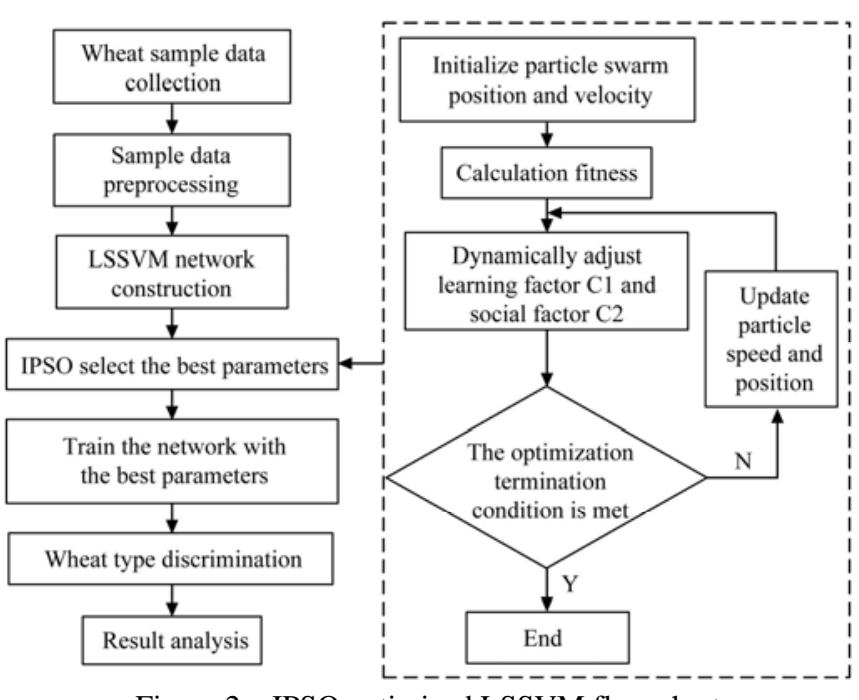

Figure 2 IPSO optimized LSSVM flow chart

\section{Results and Discussion}

\subsection{Electronic tongue response signal}

Figure 3 shows the electronic tongue response signal of a wheat sample excited by a large pulse signal at eight working electrodes. It can be clearly seen from the Figure 3 that there are large differences in the response signals of the eight working electrodes. For the sake of avoiding the complexity of the experimental data, the electrode which is as small as possible and include the overall information of the sample should be selected. After several validation experiments, it was found that the electrodes of glassy carbon, nickel, palladium, platinum, tungsten, titanium, gold and silver can basically reflect the overall information of wheat. Therefore, in this experiment, these eight precious metal electrodes were used to detect and analyze wheat of different ages. After test by electronic tongue, 8000 original data points can be obtained from each sample, and the total data matrix of $100 \times 8000$ can be obtained for five years of wheat. 


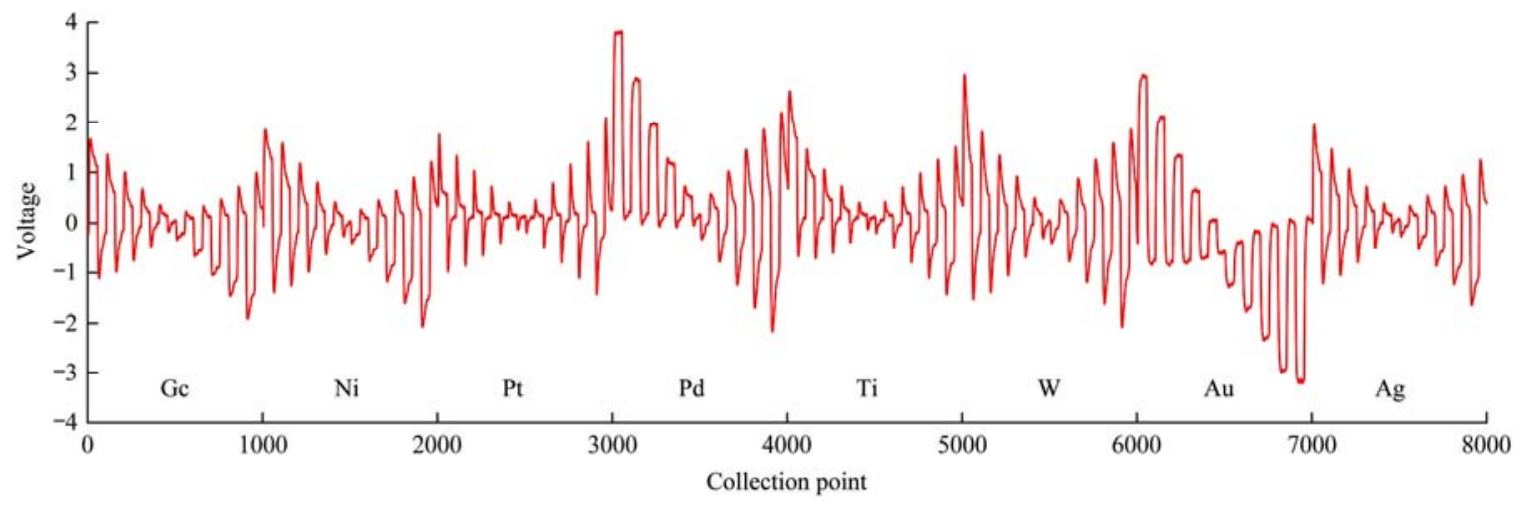

Figure 3 The response curve of electronic tongue.

\subsection{Data preprocessing}

The output signal of the electronic tongue is complex. At the same time, due to the low selectivity and interaction sensitivity of the sensor, there is a large amount of redundant information and external noise exist in the detected data ${ }^{[24]}$. Therefore, The Discrete wavelet transform (DWT) was used to extract the eigenvalues of the collected data for reducing the amount of data.

The mother wavelet and decomposition layer of DWT are two important parameters that influenced the decomposition effect of wavelet decomposition. According to the characteristics of the electronic tongue response signal, Symlets, Daubechies, Haar and Coiflets mother wavelet functions were used to decompose the electronic tongue response signals into 6-9 layers, respectively. The waveform similarity coefficient $R$ (the larger $R$ indicates the higher similarity between the two waveforms) is obtained by approximation coefficient reconstructed signal and the original signal, with the result is shown in Figure 4. It can be seen that as the number of decomposition layers increases, the similarity coefficient shows a trend of increasing and then decreasing, indicating that as the number of decomposition layers increases, more redundant information can be reduced. The increase of decomposition layers led to the reduction of the amount of data, which caused excessive loss of effective information. In order to keep the original information characteristics and obtain the optimal compression effect, the parameters with large similarity coefficient $R$ was selected. Through experimental observation, the mother wavelet of sym 4 and the decomposition layers of 8 were selected, in which the similarity coefficient $R$ is 0.9786 . After DWT compression processing, the original data was changed from $100 \times 8000$ to $100 \times 60$, which greatly reduces the amount of data.

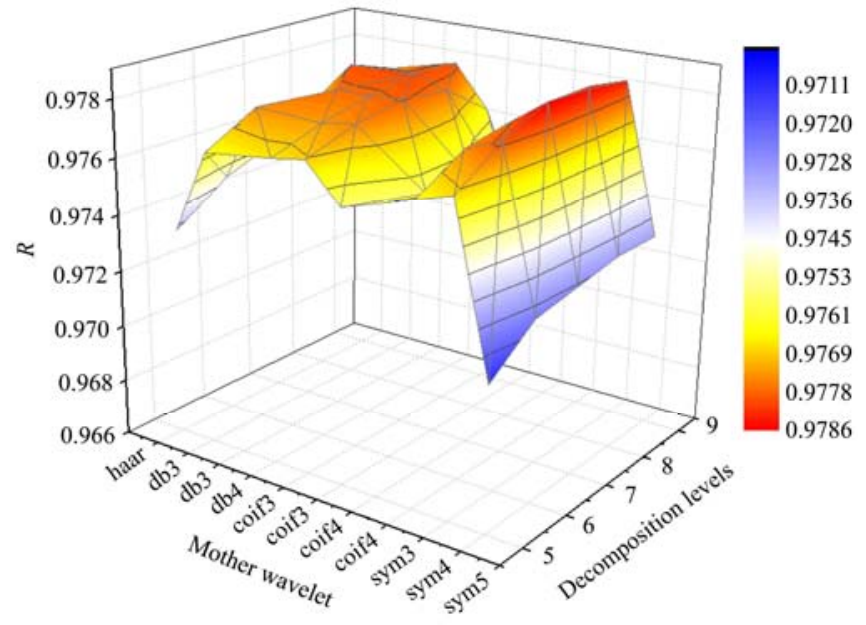

Figure 4 Parameter Optimization of DWT

\subsection{Qualitative analysis of aged grain based on DWT - IPSO - LSSVM}

In order to accurately distinguish the five storage years of aged wheat, the data processed by DWT was used as the input data of the model, and the improved particle swarm optimization algorithm optimized Least squares support vector machine model was used to make a qualitative analysis of the wheat with five storage years. The initialization parameters of improved particle swarm optimization are set as follows: The particle swarm dimension is 2, the number of particles in each particle swarm is 40 , and the maximum number of iterations is 100 . A sample of 75 (15 in each group, 5 in total) was used as the training set, and 25 (5 in each group, 5 in total) samples were used as test sets. The obtained test sample confusion matrix diagram was shown in Figure 5, in which the abscissa is the target category, the ordinate is the prediction category, and 1-5 respectively represents the aged wheat with 5 storage years. It can be seen that one sample of the three-year aged in the test sample was misclassified as a four-year aged wheat, and one sample of four-year aged wheat was misclassified as a five-year aged wheat. The rest of the samples showed no confusion, and the overall recognition rate of the test set reached 92\%. The DWT-IPSO-SVM model exhibits a good ability to differentiate aged wheat, which can effectively identify wheat of different ages.

\begin{tabular}{|c|c|c|c|c|c|c|}
\hline 1 & 5 & 0 & 0 & 0 & 0 & $\begin{array}{c}100 \% \\
0 \%\end{array}$ \\
\hline 2 & 0 & 5 & 0 & 0 & 0 & $\begin{array}{l}83.3 \% \\
16.7 \%\end{array}$ \\
\hline 3 & 0 & 0 & 4 & 0 & 0 & $\begin{array}{c}100 \% \\
0 \%\end{array}$ \\
\hline 4 & 0 & 0 & 1 & 4 & 0 & $\begin{array}{l}80 \% \\
20 \%\end{array}$ \\
\hline 5 & 0 & 0 & 0 & 1 & 5 & $\begin{array}{l}80 \% \\
20 \%\end{array}$ \\
\hline & $\begin{array}{c}100 \% \\
0 \%\end{array}$ & $\begin{array}{c}100 \% \\
0 \%\end{array}$ & $\begin{array}{l}60 \% \\
40 \%\end{array}$ & $\begin{array}{l}80 \% \\
20 \%\end{array}$ & $\begin{array}{c}100 \% \\
0 \%\end{array}$ & $\begin{array}{c}92 \% \\
8 \%\end{array}$ \\
\hline & 1 & 2 & 3 & 4 & 5 & \\
\hline
\end{tabular}

Figure 5 Sample confusion matrix of DWT-IPSO-LSSVM model

\subsection{Model verification and comparative analysis}

In order to verify the advantages of IPSO, a set of comparative experiments was carried out by using DWT-IPSO-LSSVM and DWT-PSO-LSSVM models respectively. The parameters of the traditional particle swarm optimization algorithm and the improved particle swarm optimization algorithm are same. The iterative 
convergence curves of the two algorithms are shown in Figure 6. It can be seen from the Figure 6 that the mean square error of the DWT+IPSO+LSSVM decreases rapidly in the early stage of optimization, and then the decline gradually slows down, finally tends to a constant value gradually. The iterative convergence curve of the DWT+PSO+LSSVM also decreases rapidly, then slowly declines, but the convergence speed is slower. Under the same number of iterations, the improved particle swarm optimization has a better convergence effect than the traditional particle swarm optimization algorithm.

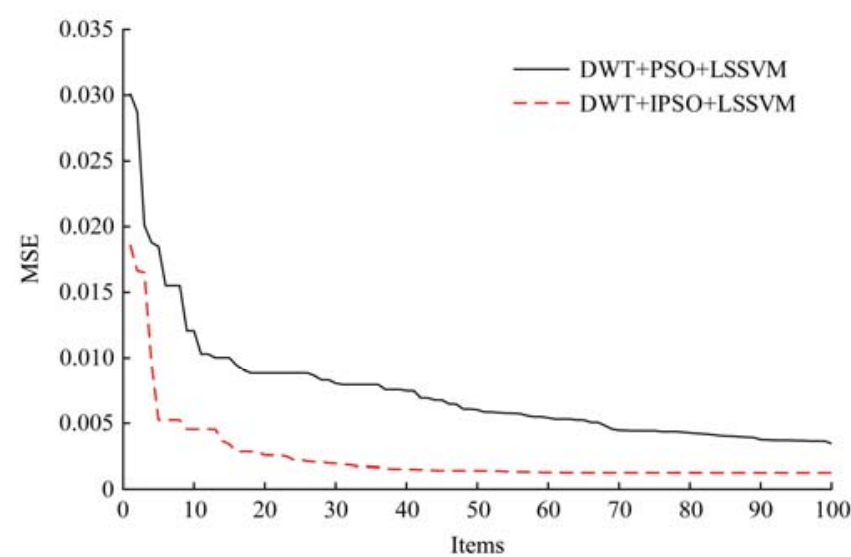

Figure 6 Iterative graph of IPSO optimization LSSVM

In order to further verify the performance of the DWT-IPSO-LSSVM model, based on the data preprocessed by DWT, the parameters of the LSSVM model were optimized by IPSO and then the wheat samples of five aging years were detected. At the same time, the classification performance were compared by the Genetic algorithm optimization Least squares support vector machine (GA-LSSVM) model and the Artificial fish swarm algorithm optimization Least squares support vector machine (AF-LSSVM) model.

The DWT- GA-LSSVM, DWT-AF-LSSVM and IPSO-LSSVM models with the same size and the maximum number of iterations are selected for comparative analysis. Based on the same data source, the model is trained by the training set. The classification performance of the model is verified by the test set. The evaluation index of the model contains the accuracy of the training set, the accuracy of the test set, the overall classification accuracy (OA) and kappa coefficient (K). Table 1 shows the results of the classification of five storage years of wheat for each model. OA represents the ratio of the number of samples correctly classified to the number of all samples. The kappa is an index for determining the degree of conformity between the classification result and the actual result. The formula of each evaluation index is as follows:

$$
\begin{gathered}
K=\frac{N \sum_{k=1}^{N} a_{k k}-\sum_{i=1}^{N} a_{k i} a_{i k}}{N^{2}-\sum_{i=1}^{N} a_{k i} a_{i k}} \\
O A=\frac{\sum_{k=1}^{N} a_{k k}}{\sum_{i, k=1}^{N} a_{i k}}=\frac{1}{n} \sum_{k=1}^{N} a_{k k}
\end{gathered}
$$

where, $N$ represents the number of categories of samples; $a_{k k}$ represents the number of correctly classified samples in each type of sample; $n$ is the total number of samples; $a_{i k}$ represents the total number of samples of the first type, and $a_{k i}$ represents the number of samples predicted to be the i-th class.

According to the classification results of several classification methods, it can be seen that different optimization algorithms have a great impact on the classification effect of LSSVM. Among the three classification models of DWT-GA-LSSVM, DWT-AFLSSVM, and DWT-IPSO-LSSVM, the DWT-GA-LSSVM processes the worst classification effect. The accuracy of the training set, the accuracy of the test set, overall classification accuracy and the values of kappa coefficients are $90 \%, 81 \%, 89 \%$, and 0.80 respectively; DWT-IPSO-LSSVM has the best classification effect. Compared with the indexes of performance of DWT-AF-LSSVM, the indexes of performance of DWT-IPSOLSSVM are increased by $2 \%, 5 \%, 1 \%$ and 0.04 on accuracy of the training set, accuracy of the test set, overall classification accuracy and kappa coefficient, respectively. This results can be explained by the poor local optimization ability of GA, which is prone to the premature phenomenon; AF has large blindness and randomness, which makes this algorithm slow to converge and easily fall into local optimum in the later stage; The proposed IPSO model is improved on the basis of PSO, which can accelerate the convergence speed in the later stage, and has better optimal solution. In addition, the classification accuracy of the imported IPSO-LSSVM model without DWT pre-processing is much lower than that of the DWT-IPSO-LSSVM model. The DWT can remove a large amount of noise and interference information, which greatly improve the operation speed and accuracy of the model.

Table 1 Model classification results

\begin{tabular}{lcccc}
\hline & $\begin{array}{c}\text { The accuracy of the } \\
\text { training set }\end{array}$ & $\begin{array}{c}\text { The accuracy } \\
\text { of the test set }\end{array}$ & OA & Kappa \\
\hline DWT-GA-LSSVM & $90 \%$ & $81 \%$ & $89 \%$ & 0.80 \\
DWT-AF-LSSVM & $93 \%$ & $87 \%$ & $90 \%$ & 0.84 \\
IPSO-LSSVM & $92.33 \%$ & $86 \%$ & $90.5 \%$ & 0.87 \\
DWT-IPSO-LSSVM & $95 \%$ & $92 \%$ & $91 \%$ & 0.88 \\
\hline
\end{tabular}

\section{Conclusion}

(1) In view of the complexity of traditional wheat detection methods, a discrimination model based on electronic tongue technology is proposed. The DWT-IPSO-LSSVM model was used to identify 5 different storage years of wheat samples. The results show that the model has a good classification effect, which can provide theoretical basis and technical support for the discrimination of aged grain.

(2) Experiments show that the classification effect of the DWT pre-processed model is significantly better than the classification model without data pre-processing. It shows that DWT pre-processing can effectively retain wheat information, eliminate noise interference information and compress the data volume of electronic tongue.

(3) In order to solve the uncertainty of the penalty factor and kernel function in the LSSVM model, an improved particle swarm optimization algorithm was proposed to optimize the LSSVM. This algorithm has the advantages of fast optimization speed, high precision, and strong ability to avoid local optimization.

\section{Acknowledgments}

Financial support for this work was provided by the Shandong Provincial Natural Science Foundation (ZR2019MF024), Top Talents Program for One Case One Discussion of Shandong Province, Science and technology development center of the Ministry of Education (2018A02010) and The Next Generation Internet Technology Innovation Project of the Sal Network (NGII20170314) and Science and Technology Development Program of Zibo (2018kj010073). 


\section{[References]}

[1] Tang W N, Liu J Y, Chen Z H, et al. Detection method of freshness adopted in grain purchasing. Gereal and feed industry, 2016, 12(10): 61-62. doi: 10.7633/j.issn.1003-6202. 2016.10.015. (in Chinese)

[2] Zhang S P. Identification of wheat aging. Food science and technology, 2012(10): 135-138. (in Chinese)

[3] Zhang H H, Wu X L, Qi M,Tang H L, Ye F K. Analysis on the present situation and New method of New maturity Identification of Wheat. Grain processing, 2016(3): 17-20. (in Chinese)

[4] Yang H P, Song W, Yuan J, Wang S Y, Pan D J. Methods of Dedermining Wheat Aging Degree. Journal of the Chinese and oils Association, 2004, 19(6): 23-26. doi: 10.3321/ j.issn:1003-0174.2004.06.007. (in Chinese)

[5] Xu M, Wang J, Zhu L Y. The qualitative and quantitative assessment of tea quality based on E-nose, E-tongue and E-eye combined with chemometrics. Food chemistry, 2019, 289: 482-489. doi: 10.1016/ j.foodchem.2019.03.080

[6] Winquist F, Olsson J, Eriksson M. Multicomponent analysis of drinking water by a voltammetric electronic tongue. Analytica Chimica Acta, 2011, 683(2): 192-197. doi: 10.1016/j.aca.2010.10.027

[7] Xavier Cetó, AndreuGonzález-Calabuig, NoraCrespo, Sandra Pérez and M. D. Valle. Electronic tongues to assess wine sensory descriptors. Talanta, 2017(162): 218-224. doi: 10.1016/j.talanta.2016.09.055.

[8] Robertoh L, Rafael M, Miguel A, et al. Prediction of $\mathrm{NaCl}$, nitrate and nitrite contents in minced meat by using a voltammetric electronic tongue and an impedimetric sensor. Food Chemistry, 2010, 122(3): 864-870. doi: 10.1016/j.foodchem.2010.02.04.

[9] Lu L, Hu X Q, Tian S Y, Deng S P, Zhu Z W. Visualized attribute analysis approach for characterization and quantification of rice taste flavor using electronic tongue. Analytica chimica acta, 2016, 919: 11-19. doi: 10.1016/j.aca.2016.03.019

[10] Liu S Y, Xu L Q, Li Z B, Li D L. Forecasting Model for pH Value of Aquaculture Water Quality Based on PCA-MCAFA-LSSVM. Transactions of the Chinese Society for Agricultural Machinery 2014, 45(5): 239-246. doi: 10.6041/j.issn.1000-1298.2014.05.037. (in Chinese)

[11] $\mathrm{Lu} \mathrm{L,} \mathrm{Hu} \mathrm{X,} \mathrm{Tian} \mathrm{S,} \mathrm{Deng} \mathrm{S,} \mathrm{Zhu} \mathrm{Z.} \mathrm{Visualized} \mathrm{attribute} \mathrm{analysis}$ approach for characterization and quantification of rice taste flavor using electronic tongue. Analytica Chimica Acta, 2016, 919: 11-19. doi: 10.1016/j.aca.2016.03.019.

[12] Wei Z B, Yang Y N, Wang J, Zhang W L, Ren Q F. The measurement principles, working parameters and configurations of voltammetric electronic tongues and its applications for foodstuff analysis. Journal of food engineering, 2018, 217: 75-92. doi: 10.1016/j.jfoodeng.2017.08.005.

[13] Tian X J, Wang J, Zhang X. Discrimination of preserved licorice apricot using electronic tongue. Mathematical \& Computer Modelling, 2013,
58(3-4): 737-745. doi: 10.1016/j.mcm.2012.12.034.

[14] Wei Z B, Wang J, Zhang X. Monitoring of quality and storage time of unsealed pasteurized milk by voltammetric electronic tongue. Electrochimica Acta, 2013, 88(2): 231-239. doi: 10.1016/ j.electacta.2012.10.042

[15] Liu M, Wang M J, Wang J, Li D. Comparison of random forest, support vector machine and back propagation neural network for electronic tongue data classification: Application to the recognition of orange beverage and Chinese vinegar. Sensors and Actuators B: Chemical, 2013, 177: 970-980. doi: 10.1016/j.snb.2012.11.071.

[16] Qiu S S, Wang J, Tang C, Du D D. Comparison of ELM, RF, and SVM on E-nose and E-tongue to trace the quality status of mandarin (Citrus unshiu Marc.). Journal of food engineering, 2015, 166: 193-203. doi:10.1016/j.jfoodeng.2015.06.007

[17] Ma Z L, Yin T J, Guo T T,Wang Z Q, Li C H, Guo Y M. The brand and purity of orange juice and liquor were tested by electronic tongue method. Science and Technology of Food Industry, 2018(8). (in Chinese)

[18] Shi Q R, Guo T T, Yin T J, Wang Z Q, Li C H, Sun X, et al. Classification of Pericarpium Citri Reticulatae of Different Ages by Using a Voltammetric Electronic Tongue System. International Journal Of Electrochemical Science, 2018, 13(12): 11359-11374. doi: 10.20964/ 2018.12.45

[19] He X, Wang H L, Lu J H, Jiang W. Analog circuit fault diagnosis method based on preferred wavelet packet and ELM. Chinese Journal of Scientific Instrument, 2013, 34(11): 2614-2619. doi: 10.3969/ j.issn.0254-3087.2013.11.030. (in Chinese)

[20] Yu H H, Chen Y Y, Hassan S G, Li D L. Prediction of the temperature in a Chinese solar greenhouse based on LSSVM optimized by improved PSO. Computers and Electronics in Agriculture, 2016, 122: 94-102. doi: 10.1016/j.compag.2016.01.019.

[21] Shi Q R. Research on food and drug analysis based on electronic tongue and pattern recognition technology. Shandong University of Technology, 2018. (in Chinese)

[22] Fernandez-Martinez J L, Garcia-Gonzalo E. Stochastic stability analysis of the linear continuous and discrete PSO models. IEEE Transactions on Evolutionary Computation, 2011, 15(3): 405-423. doi: 10.1109/ TEVC.2010.2053935.

[23] Firouzib B, Sadeghi M S, Niknam T. A new hybrid algorithm based on PSO, SA, and K-means for cluster analysis. International Journal of Innovative Computing Information and Control, 2010, 6(7): 3177-3192.

[24] Shi Q R, Ma Z L, Zhou Z, He X Q, Wang Z Q, Ma Y X. Research on Chinese patent medicine identification method based on electronic tongue technology and pattern recognition. Journal of Electronic Measurement and Instrumentation, 2017, 31(7): 1081-1089. doi: 10.13382/ j.jemi.2017.07.014. (in Chinese) 